\title{
MedienPädagogik
}

Zeitschrift für Theorie und Praxis der Medienbildung

Themenheft Nr. 29: Die Konstitution der Medienpädagogik. Zwischen interdisziplinärem Forschungsfeld und bildungswissenschaftlicher (Sub-) Disziplin.

Hrsg. v. Christian Swertz, Wolfgang B. Ruge, Alexander Schmölz und Alessandro Barberi.

\section{Diskurs als konstituierende Grundlage der Medienpädagogik und Folgerungen aus systemtheoretischer Sicht}

Gerhard Tulodziecki

\begin{abstract}
Zusammenfassung
Medienpädagogik lässt sich beschreiben als Wissenschaft und Lehre von medienbezogenen erziehungs- und bildungsrelevanten Aktivitäten und ihren Voraussetzungen und Bedingungen. Vielfältige Entwürfe und Diskussionslinien in der Medienpädagogik haben bisher weder zu einer allgemein anerkannten Theorie mit einem eindeutig umgrenzten Gegenstand noch zu einer spezifischen Forschungsmethodik geführt. Vor diesem Hintergrund wird in dem vorliegenden Beitrag der medienpädagogische Diskurs selbst als konstituierende Grundlage der Medienpädagogik verstanden. Als Themenfelder des Diskurses lassen sich u. a. nennen: begriffliche Klärungen sowie Voraussetzungen, Bedingungen, Zielvorstellungen mit ihren Inhalten und Vorgehensweisen für erziehungs- und bildungsrelevante Aktivitäten mit Medienbezug sowie Forschungsmethoden (einschliesslich jeweiliger Begründungen). Mit Blick auf einen so strukturierten medienpädagogischen Diskurs geht es des Weiteren um Überlegungen a) zur Umwandlung von Informationen des Diskurses in ein (personengebundenes) Reflexions- und Gestaltungswissen für erziehungs- und bildungsbezogenes Handeln, b) zum Verhältnis von Theorie und Praxis als reflexives und gestaltungsorientiertes In-Beziehung-Setzen von Diskursthemen und pädagogisch relevanten Situationen und Prozessen sowie c) zu Möglichkeiten und Problemen einer Begrenzung des medienpädagogischen Diskurses zur Reduzierung von Komplexität bei gleichzeitiger Öffnung für Weiterentwicklungen.
\end{abstract}

Discourse as a Constituent Basis of Media Pedagogy and Conclusions from a Perspective of Systems Theory

\begin{abstract}
Media Pedagogy can be described as the theory of media education and media literacy activities and their preconditions and circumstances. Various drafts and lines of discussion in the discourse on media pedagogy have neither led to a generally accepted theory with a clearly limited object nor to specific research methods. Against this background, the discourse on media pedagogy itself will be understood as a constituent basis of media
\end{abstract}


pedagogy in the following paper. Topics of this discourse include conceptual clarifications as well as preconditions, circumstances, aims and procedures of media education and media literacy activities and research methods. With reference to the discourse structured this way, this article, moreover, deals with considerations a) on converting information of the media pedagogical discourse to (personal) reflexive and design knowledge and competencies for educational actions, b) on the relation between theory and practice as a reflexive and design-oriented relating of topics of the discourse with pedagogically relevant situations and processes, and c) on possibilities and problems of limiting the media pedagogical discourse in order to reduce complexity and of opening it for further developments at the same time.

\section{Einleitung: Zum Begriff der Medienpädagogik}

Der Begriff der Medienpädagogik wird im allgemeinen Sprachgebrauch unterschiedlich verwendet. So sagt man gegebenenfalls sowohl von Eltern, die mit ihren Kindern eine Vereinbarung über die Nutzung des Smartphones treffen, als auch von Wissenschaftlerinnen und Wissenschaftlern, die eine Untersuchung zu Effekten der Computernutzung in der Schule durchführen, dass sie Medienpädagogik betreiben. In seiner allgemeinsten Form umfasst der Begriff der Medienpädagogik - nicht zuletzt als Folge der Mehrdeutigkeit des Pädagogikbegriffs - sowohl erziehungs- und bildungsrelevante Verhaltensweisen und Handlungen in Medienzusammenhängen als auch Erwägungen und Äusserungen dazu. Anders ausgedrückt: Medienpädagogik wird umgangssprachlich sowohl für praktisches Verhalten und Handeln samt zugehörigen Überlegungen als auch für wissenschaftliche Reflexionen und Aktivitäten genutzt.

Da es in diesem Beitrag um die Konstitution von Medienpädagogik geht, empfiehlt es sich, den Begriff gegenüber einem umgangssprachlichen Verständnis einzugrenzen: Er soll hier und im Folgenden als Wissenschaft und Lehre von erziehungsund bildungsrelevanten Aktivitäten mit Medienbezug und ihren Voraussetzungen und Bedingungen verstanden werden. Mit dem Definitionsmerkmal «Wissenschaft und Lehre» wird hervorgehoben, dass der Medienpädagogik - in dem hier zugrunde liegenden Verständnis - die Aufgabe zukommt, auf wissenschaftlicher Grundlage ein zusammenhängendes Gefüge von Aussagen bzw. Sätzen zu formulieren, das zum einen auf Erkenntnisgewinn zielt und zum anderen in der Aus-, Fort- und Weiterbildung für pädagogische Tätigkeiten zur Geltung kommt und dabei Anregung und Unterstützung für pädagogisch tätige Personen bietet. Die Gegenstandsbeschreibung "erziehungs- und bildungsrelevante Aktivitäten mit Medienbezug» soll verdeutlichen, dass nur solche Aktivitäten Gegenstand der Medienpädagogik sind, die Erziehungs- oder Bildungsrelevanz besitzen und gleichzeitig einen Medienbezug haben, wobei das Merkmal «Medienbezug» sowohl Situationen umfasst, in denen Medien als Mittel oder Instrument verwendet werden, als auch Situationen, in denen es um eine 
inhaltliche Auseinandersetzung mit oder eine Reflexion zu Medienfragen geht. Der Definitionsverweis auf «Voraussetzungen und Bedingungen» bedeutet darüber hinaus, dass stets auch der Kontext zu beachten ist, in dem erziehungs- und bildungsrelevante Aktivitäten mit Medienbezug stehen - seien es personale, technologische, rechtliche, ökonomische, institutionelle, politische oder andere historische, kulturelle und gesellschaftliche Voraussetzungen und Bedingungen. Insgesamt soll Medienpädagogik sowohl der Aufklärung erziehungs- und bildungsrelevanten medienbezogenen Tuns als auch seiner Gestaltung dienen. Insofern wird Medienpädagogik als Reflexions- und Handlungswissenschaft verstanden.

Fragt man nach der Konstitution einer solchen Medienpädagogik, so ist der Fokus zunächst auf ihren Ursprung zu richten. Dieser ist darin zu sehen, dass Überlegungen zu medienbezogenen Aktivitäten unter einer Erziehungs- und Bildungsperspektive sprachlich formuliert werden und im Sinne von Wissenschaft und Lehre Geltung beanspruchen. Dies wiederum führt dazu, dass die sprachlich gefassten Überlegungen Eingang in Diskurse finden und dort gegebenenfalls zu Kritik und Gegenkritik und/ oder zu ergänzten, erweiterten oder alternativen Entwürfen führen (vgl. zu solchen Prozessen und zur diesbezüglichen Rolle von Medien z. B. Swertz 2008).

Blickt man auf die zahlreichen Entwürfe zur Medienpädagogik, so muss man zunächst feststellen, dass sich weder eine allgemein anerkannte Theorie mit einem eindeutig umrissenen Gegenstand noch ein generell zugrunde liegendes Forschungsverfahren herausgebildet haben (was im Übrigen ebenso für andere Disziplinen gilt, vgl. dazu z. B. Benner 2012, 15). Auch die oben verwendete allgemeine Gegenstandsbeschreibung, dass es in der Medienpädagogik um «erziehungs- und bildungsrelevante Aktivitäten mit Medienbezug und ihre Voraussetzungen und Bedingungen» geht, lässt wegen unterschiedlicher Verständnisse von Erziehung, Bildung und Medien sowie zahlreicher Voraussetzungen und Bedingungen vielfältige Interpretationen zu. Am ehesten könnte noch das Merkmal der Erziehungs- und Bildungsperspektive als ein Unterscheidungskriterium zu anderen Disziplinen oder Teildisziplinen dienen, z.B. zur Kommunikationswissenschaft, Medienpsychologie oder Mediensoziologie. Allerdings ist auch bezüglich dieses Unterscheidungskriteriums zu bedenken, dass Erziehung und Bildung eng mit Fragen von Kommunikation und Lernen, Entwicklung und Sozialisation verbunden sind, sodass mit der Erziehungs- und Bildungsperspektive zwar eine spezifische Sichtweise auf medienbezogene Aktivitäten besteht, aber keine völlig trennscharfe Abgrenzung erreicht wird.

Die bisherigen Überlegungen lassen es geraten erscheinen, den medienpädagogischen Diskurs nicht als (vergebliche) Suche nach einem exklusiven Gegenstand oder einer exklusiven Forschungsmethode der Medienpädagogik aufzufassen, sondern den Diskurs selbst als konstituierende Grundlage der Medienpädagogik zu begreifen. Dabei gilt allerdings, dass ein Diskurs an sich noch kein genuines Merkmal der Medienpädagogik ist. Auch für andere Disziplinen können Diskurse eine konstituierende 
Bedeutung haben. Eine Unterscheidung zu anderen Disziplinen ergibt sich jedoch durch die Verbindung von Diskurs und den - auf erziehungs- und bildungsrelevante Aktivitäten mit Medienbezug im obigen Sinne gerichteten - Fragestellungen bzw. Inhalten.

Vor diesem Hintergrund wird der medienpädagogische Diskurs im Folgenden zunächst hinsichtlich seiner Themenfelder und Fragestellungen charakterisiert. Anschliessend geht es um Überlegungen, a) wie der Diskurs zu Reflexions- und Gestaltungswissen für Erziehungs- und Bildungssituationen führen kann, b) wie sich vor diesem Hintergrund das Verhältnis von Theorie und Praxis beschreiben lässt und c) wie sich das Problem der Balance zwischen Grenzziehung und Öffnung zu verwandten Diskursen aus systemtheoretischer Sicht darstellt. Entsprechende Fragen gelten nicht für die Medienpädagogik allein, sondern auch für andere Teildisziplinen der Erziehungswissenschaft oder für vergleichbare Wissenschaften. Das heisst aber zugleich, dass sich auch die Medienpädagogik mit ihren spezifischen Inhalten bzw. Blickrichtungen den entsprechenden Fragen stellen muss.

\section{Der medienpädagogische Diskurs mit seinen Themenfeldern als konstituierende Grundlage}

Mit den bisherigen Überlegungen wird betont, dass Medienpädagogik als Disziplin nicht einfach mit ihrem Gegenstand oder einer spezifischen Forschungsmethode vorgegeben ist, sondern erst durch den medienpädagogischen Diskurs hervorgebracht wird (vgl. zur diesbezüglichen Bedeutung von Diskursen u. a. Foucault 1981). Der medienpädagogische Diskurs realisiert sich dabei vor allem in Vorträgen und Diskussionen bei Tagungen medienpädagogischer Vereinigungen, z. B. der DGfE-Sektion Medienpädagogik oder der Gesellschaft für Medienpädagogik und Kommunikationskultur (GMK), in Sammelbänden, z. B. in den Jahrbüchern Medienpädagogik oder in Handbüchern zur Medienpädagogik, in Monographien, in denen unterschiedliche medienpädagogische Positionen zur Sprache kommen, sowie in Zeitschriften, z. B. in der Zeitschrift merz medien + erziehung sowie in den Online-Zeitschriften MedienPädagogik und medienimpulse.

Im Diskurs treffen unterschiedliche Auffassungen zu gegebenen oder wünschenswerten Aktivitäten mit Medienbezug unter der Erziehungs- und Bildungsperspektive aufeinander. Die unterschiedlichen Auffassungen spiegeln sich zum einen in der Auseinandersetzung mit Einzelfragen wider, z. B. wie der Begriff der Medienkompetenz zu verstehen sei (vgl. u. a. Spanhel 2006, 212-218; Tulodziecki 2015, 195-210), oder in der Differenz umfassenderer Entwürfe, z. B. zur Medienbildung (vgl. u. a. Jörissen und Marotzki 2009; Tulodziecki, Herzig und Grafe 2010).

Generell kann ein Diskurs unterschiedliche Themen aufgreifen (vgl. z. B. Budde 2012). So lassen sich auch im medienpädagogischen Diskurs bestimmte Themenfelder 
ausmachen (vgl. z. B. Aßmann et al. 2016; Bettinger und Aßmann 2017). Dabei treten hinsichtlich einer Gliederung unterschiedliche Möglichkeiten zutage. So gibt es unter anderem Diskurse zu Teilbereichen, z. B. zur Mediendidaktik, zur Medienerziehung und zur Medienbildung, oder zu wissenschaftsimmanenten Fragen, z.B. zu Begriffsdefinitionen, zum Stellenwert normativer oder empirischer Sätze, zum Theorieverständnis und zu geeigneten Forschungsmethoden (vgl. z. B. Sesink et al. 2007). Eine Gliederung des Diskurses nach Teilbereichen hätte zwar den Vorzug eines spezifischeren Zugangs zu einzelnen Aufgabenfeldern, z. B. Lernen mit Medien versus Lernen über Medien, könnte jedoch dazu führen, dass Zusammenhänge zwischen den verschiedenen Teilbereichen sowie übergreifende Fragen der Medienpädagogik vernachlässigt würden. Deshalb wird der Diskurs im Folgenden nach Fragen strukturiert, die für alle Teilbereiche relevant sind. Dazu wähle ich Themenfelder, die im Hinblick auf die Theoriebildung und das Methodenbewusstsein der Medienpädagogik als Wissenschaft besonders wichtig sind. In diesem Zusammenhang erfolgt eine Orientierung an einem Wissenschaftsverständnis, nach dem Medienpädagogik als Handlungs- und Reflexionswissenschaft aufgefasst wird und sowohl empirische und logische als auch normative und handlungsanleitende Sätze umfassen darf und muss (vgl. zu einem solchen Wissenschaftsverständnis z. B. Tulodziecki et al. 2013, 99-101).

Demgemäss kann der medienpädagogische Diskurs - ohne Anspruch auf Vollständigkeit - durch die nachstehenden Inhalte bzw. Themenfelder und Fragestellungen strukturiert werden. Da sich entsprechende Diskursbeiträge auf viele Tagungen und Veröffentlichungen verteilen, könnten jeweils umfangreiche Literaturlisten angeführt werden. Im Rahmen dieses Aufsatzes beschränke ich mich jedoch auf einzelne Literaturangaben zu Sammelbänden oder Übersichtswerken, in denen wichtige Beiträge zum angesprochenen Themenfeld und so auch weitere Literaturhinweise zu finden sind:

- Begriffsdefinitionen: Wie sollen z. B. die Begriffe Medium oder Medialität, Kompetenz oder Bildung, Erziehung oder Sozialisation, Lernen oder Entwicklung, Handeln oder Kommunikation im Rahmen der Medienpädagogik gefasst werden? Welche Gründe sprechen für bestimmte definitorische Entscheidungen? (Vgl. z. B. Hüther und Schorb 2005; Sander et al. 2008.)

- Zentrale Konstrukte: Welche Konstrukte sollen als Ausgangspunkte für medienpädagogische Überlegungen dienen und gegebenenfalls eine Leitfunktion übernehmen, z. B. Erziehung, Entwicklung, Kommunikation, Kompetenz, Bildung, Aneignung, Handeln und/oder Kultur? Welche Begründungen ergeben sich für zentrale Ausgangspunkte oder Leitbegriffe? Wie ist gegebenenfalls das Verhältnis der Konstrukte zueinander zu konzipieren? (Vgl. z. B. Moser, Grell und Niesyto 2011.)

- Individuelle Voraussetzungen und Bedingungen für Lern-, Erziehungs- oder Bildungsprozesse in Medienzusammenhängen: Mit welchen Voraussetzungen ist z.B. 
bei Kindern und Jugendlichen für entsprechende Prozesse zu rechnen und welche Unterschiede sind diesbezüglich zu erwarten? Welche Erklärungen liegen gegebenenfalls vor? Welche Bedingungen müssen dabei beachtet werden? Welche Kompetenz ist bei pädagogisch Agierenden vorhanden und welche ist erforderlich? Haben sich entsprechende Annahmen bereits bewährt? Wie lässt sich ihre Richtigkeit gegebenenfalls sichern? (Vgl. z. B. von Gross et al. 2015; Gysbers 2008; Schulz-Zander et al. 2012.)

- Zielvorstellungen mit ihren Inhalten für medienbezogene Aktivitäten: Welches Wissen und Können, welche Haltungen und Einstellungen, welche Verhaltens- und Handlungsweisen sind aus erzieherischer und bildungsbezogener Sicht anzustreben? Auf welche medienbezogenen Inhalts- und Nutzungsbereiche sollen sich Wissen und Können beziehen? Stellen z. B. Medienkompetenz oder Medienbildung geeignete Zielkategorien dar und wie lassen sie sich gegebenenfalls für praktisches Vorgehen operationalisieren? Wie können bestimmte Zielvorstellungen begründet werden? Welches Menschenbild ist mit den Zielvorstellungen und Begründungen verbunden? (Vgl. z. B. Herzig et al. 2010.)

- Vorgehensweisen für Lern-, Erziehungs- und Bildungsprozesse in Medienzusammenhängen: Wie können ziel- und entwicklungsgemässe Prozesse angeregt und unterstützt werden? Welche Lernschritte oder Lernphasen erscheinen besonders geeignet? Wie ist das Verhältnis von gestaltend-produktorientierten und analytisch-reflektierenden, von individuellen und sozialgebundenen, von mediengestützten und personalen, von exemplarischen und orientierenden Vorgehensweisen zu konzipieren? Welche empirischen Annahmen zu Lern-, Lehr- und Entwicklungsprozessen liegen dabei zugrunde? (Vgl. z.B. Tulodziecki, Herzig u. Grafe 2010.)

- Institutionelle Voraussetzungen und Bedingungen für Lern-, Erziehungs- und Bildungsprozesse mit Medienbezug: Welche technischen, rechtlichen, ökonomischen, personalen und weiteren gesellschaftlichen Voraussetzungen und Bedingungen sind für die Institutionen gegeben, in deren Rahmen medienbezogene Lern-, Erziehungs- und Bildungsprozesse angeregt und unterstützt werden oder werden sollen? Welche Erklärungsansätze können für diagnostizierte Zustände herangezogen werden? Welche Voraussetzungen und Bedingungen sind mit welchen Begründungen erforderlich und wie können gegebenenfalls Verbesserungen erreicht werden? (Vgl. z. B. Eickelmann 2010; Breiter et al. 2010.)

- Forschungsvorgehen: Welche Forschungsmethoden eignen sich zur Bearbeitung der unterschiedlichen Fragestellungen? Bei welchen Fragestellungen sind z. B. eher empirische, eher historisch-hermeneutische, eher ideologiekritische oder eher strukturanalytische Vorgehensweisen angebracht? Welche Mischformen bieten sich gegebenenfalls an? In welchem Verhältnis sollen dabei quantitativ und qualitativ orientierte Vorgehensweisen stehen? (Vgl. z. B. Hartung et al. 2014.) 
Fasst man den medienpädagogischen Diskurs - gemäss obigen Überlegungen - als konstituierende Grundlage der Medienpädagogik auf, lässt sich mit Bezug auf die genannten Diskursfelder auch sagen, dass die Medienpädagogik durch diese und ihren Zusammenhang konstituiert wird.

\section{Vom medienpädagogischen Diskurs zu medienpädagogischem Wissen}

In diesem Abschnitt gehe ich der Frage nach, wie eine - gemäss obigen Überlegungen - konstituierte Medienpädagogik zu wirksamem Wissen für die Reflexion und Gestaltung von Erziehungs- und Bildungssituationen mit Medienbezug führen kann. Strukturell geht es dabei um das Verhältnis zwischen einem Diskurs und personalem Wissen. Für die Aufklärung dieses Verhältnisses ziehe ich im Folgenden systemtheoretische Überlegungen heran.

In Anlehnung an die soziologisch orientierte Systemtheorie von Luhmann (1984) kann der medienpädagogische Diskurs als ein soziales System aufgefasst werden. Elemente sozialer Systeme sind nach Luhmann (1984, 191-241) Kommunikationsereignisse (und nicht die agierenden Personen, die Luhmann als psychische Systeme der Umwelt sozialer Systeme zuordnet). Aus dieser Sicht stellt sich der medienpädagogische Diskurs als eine Folge von Kommunikationsereignissen dar. Jedes Kommunikationsereignis lässt sich dabei als Einheit eines dreistelligen Selektionsprozesses von Information, Mitteilung und Verstehen deuten: die erste Selektion besteht darin, etwas überhaupt als Information zu betrachten, die zweite Selektion ergibt sich durch die Entscheidung, die Information in einer bestimmten Form mitzuteilen, die dritte Selektion erfolgt dadurch, dass die Mitteilung bezüglich ihrer Information wahrgenommen wird. Alle Selektionen hätten auch anders verlaufen können. Eine agierende Person (in systemtheoretischer Formulierung: ein psychisches System) hätte auch etwas anderes als Information auswählen können, sie hätte auch auf die Mitteilung verzichten und etwas anderes tun können und schliesslich hätte die Mitteilung bzw. die Information negiert werden können. Demgemäss sind Kommunikationsereignisse durch Kontingenz gekennzeichnet, was bedeutet, dass bei jeder Selektion Alternativen möglich gewesen wären, aber nicht zum Zuge gekommen sind. Aus dieser Sicht erscheint der medienpädagogische Diskurs als ein Prozess, in dem ständig eine Auswahl aus unterschiedlichen Möglichkeiten geschieht: von einer agierenden Person werden Informationen ausgewählt und in ausgewählte Formen der Mitteilung gebracht, andere Personen richten ihre Aufmerksamkeit auf die Mitteilungen oder auf irgendetwas anderes, sie entnehmen den Mitteilungen die jeweilige Information oder unterlassen es. Insofern stellt sich der medienpädagogische Diskurs nicht als Prozess der Wissensübertragung, sondern als Selektionsprozess dar, der wegen seiner Kontingenz stets auch anders verlaufen könnte. 
Auf der Grundlage einer solchen Deutung des medienpädagogischen Diskurses ergeben sich verschiedene Folgerungen bzw. Überlegungen hinsichtlich der Entwicklung personalen medienpädagogischen Wissens:

1. Um personales Wissen zu konstruieren, müssen Personen den im medienpädagogischen Diskurs kommunizierten Mitteilungen Information entnehmen bzw. diese verstehen und in ihrer kognitiven Struktur verankern. Für entsprechende Prozesse ist es notwendig, dass die Personen der zunächst nur potenziellen Bedeutung von Informationen eine aktuelle Bedeutung zuweisen (vgl. dazu z.B. Ausubel 1978, 58-66; Tulodziecki et al. 2017, 45-52). Solche Prozesse sind dann allerdings nicht mehr Gegenstand einer soziologischen Systemtheorie, sondern der Psychologie bzw. einer personalen Systemtheorie (vgl. z. B. König und Volmer 2008).

2. Die - für die Bedeutungszuweisung relevante individuelle - kognitive Struktur lässt sich als individuelle Theorie zu erziehungs- oder bildungsrelevanten Situationen oder Vorgängen mit Medienbezug auffassen (wobei ein zunächst unspezifischer Theoriebegriff zugrunde liegt). Der individuellen Theorie steht dann der medienpädagogische Diskurs mit dem Anspruch wissenschaftlicher Theoriebildung gegenüber, wobei dieser Anspruch auf eine kohärente Zusammenstellung von intersubjektiv nachvollziehbaren Aussagen bzw. Sätzen gerichtet ist (vgl. u. a. Tulodziecki, Grafe und Herzig 2013, 21-24).

3. Das «Gegenüber» von individueller Theorie und medienpädagogischem Diskurs gilt grundsätzlich sowohl für praktisch Handelnde im Feld der Medienbildung als auch für medienpädagogisch Forschende (weil der medienpädagogische Diskurs als soziales System gemäss dem Luhmann'schen Verständnis als Folge von Kommunikationsereignissen und nicht als Gefüge von Personen zu verstehen ist).

4. Der Unterschied zwischen Praktizierenden und Forschenden besteht nicht darin, dass die einen dem medienpädagogischen Diskurs zuzurechnen sind und die anderen ihn nur rezipieren (sollen), sondern darin, dass ihre jeweiligen individuellen Theorien eine grössere Ferne oder Nähe zum Diskurs aufweisen, dass sie geringere oder bessere Chancen haben, eigene Kommunikationsbeiträge in den Diskurs einzubringen, und dass sie gegebenenfalls an unterschiedlichen Konsequenzen einer Auseinandersetzung mit dem medienpädagogischen Diskurs interessiert sind. So mag Praktizierenden vor allem daran gelegen sein, Anregungen und Unterstützung für ihre praktische Erziehungs- und Bildungsarbeit zu erhalten, während es Forschenden darauf ankommt, auf offene Fragen zu stossen, die Anlass für weitere Forschungen sein können.

5. Sowohl praktisch Handelnde als auch medienpädagogisch Forschende können vom medienpädagogischen Diskurs oder Teilen davon nur profitieren, wenn in ihren jeweils individuellen Theorien Anschlussmöglichkeiten vorhanden sind, 
z. B. bezüglich vorhandener begrifflicher Verständnisse sowie eigener Vorstellungen zu Voraussetzungen, Zielen und geeigneten Vorgehensweisen.

Diese Überlegungen liefern zugleich Hinweise auf individuelle Bedingungen, welche die von Luhmann beschriebenen Selektionsprozesse bei Kommunikationsereignissen beeinflussen. Neben individuellen Bedingungen spielen bei Selektionsprozessen innerhalb medienpädagogischer oder anderer disziplinbezogener Diskurse allerdings auch strukturelle und institutionelle Bedingungen der Wissenschaftskommunikation eine Rolle, z. B. hierarchische Verhältnisse zwischen den am Diskurs Beteiligten, Karrieremöglichkeiten oder Modalitäten der Verteilung bzw. Vergabe von Forschungsmitteln. Solche Bedingungen können hier allerdings nur angedeutet werden (vgl. zu weiteren Hinweisen u. a. Schurz 2014, 59).

Gemäss den Akzentsetzungen dieses Beitrags werden die obigen schlussfolgernden Überlegungen im nächsten Abschnitt mit der Frage weitergeführt, wie sich ein produktives Verhältnis zwischen der Praxis und dem medienpädagogischen Diskurs mit seinem Anspruch auf wissenschaftliche Theoriebildung ergeben kann.

\section{Zum Verhältnis von medienpädagogischem Diskurs und Praxis}

Bei der Frage nach dem Verhältnis von Theorie und Praxis wird in sprachlicher Hinsicht in der Regel nicht auf eine Unterscheidung zwischen individueller und wissenschaftlicher Theorie zurückgegriffen, sondern auf eine Unterscheidung nach subjektiver und wissenschaftlicher Theorie. Für subjektive Theorien gilt die Annahme, dass sie häufig aus «alltagstheoretischen» Vorstellungen erwachsen und in der Regel nicht präzise ausformuliert bzw. mehrdeutig, inkohärent und inkonsistent sind (vgl. Patry 2012 , 11). Subjektive Theorien - bei König und Volmer $(2008,141)$ auch als subjektive Deutungen bezeichnet - werden im Kontext einer personalen Systemtheorie den praktisch handelnden Personen zugeschrieben. Sie lassen sich in Anlehnung an König und Volmer $(2008,139-164)$ durch Merkmale folgender Art kennzeichnen:

- Subjektive Konstrukte: Damit sind begriffliche Vorstellungen gemeint, die als relevant eingeschätzt und zur Diagnose und Erklärung einer Situation sowie für die Beschreibung und Erwägung von Zielen und Handlungsstrategien herangezogen werden. Beispielsweise mag eine Lehrperson, die einen Unterricht zum Thema Computerspiele plant, unter anderem mit den Konstrukten «Spielerfahrungen der Lernenden», «Sensibilität für Einflüsse von Computerspielen» oder «Bereitschaft, sich mit Jugendschutz-Bestimmungen auseinanderzusetzen» operieren.

- Subjektive Diagnosen: Diese zielen auf die Zustandserfassung und enthalten häufig auch wertende Elemente. Die Lehrperson könnte im obigen Beispiel bezüglich der Ausgangssituation unter anderem zu Einschätzungen folgender Art kommen: Die Schüler verfügen zwar über vielfältige Spielerfahrungen, haben ihr Spielverhalten bisher jedoch kaum reflektiert./ Die Lernenden zeigen nur geringe 
Sensibilität für Einflüsse von Computerspielen./ Die Jugendlichen zeigen wenig Bereitschaft, sich über das Spielen hinaus auf eine kritische Reflexion einzulassen.

- Subjektive Erklärungen: Dabei geht es um Vermutungen zu der Frage, welche Ursachen für bestimmte Zustände oder Situationen anzunehmen sind. So könnte die Lehrperson in unserem Beispiel etwa meinen: Weil die Lernenden keine oder nur unzureichende Kenntnisse zu Einflüssen von Computerspielen und zu den mit ihnen verbundenen Jugendschutz-Bestimmungen haben, zeigen sie wenig Bereitschaft zu einer Reflexion.

- Subjektive Ziele: Diese stellen Zielvorstellungen dar, die als wichtig empfunden werden. Beispielsweise mag die Lehrperson bezüglich des Spielens am Computer anstreben, dass die Lernenden Kenntnisse zu Einflüssen von Computerspielen und zu rechtlichen Bedingungen der Produktion und Verbreitung von Computerspielen erwerben und damit gleichzeitig die Bereitschaft erlangen, ihr eigenes Spielverhalten kritisch zu reflektieren. Gegebenenfalls können solche Zielvorstellungen auch durch gesellschaftliche Anforderungen, wie sie z.B. in Lehrplänen formuliert sind, beeinflusst werden.

- Subjektive Strategien: Mit ihnen werden Annahmen darüber bezeichnet, welche Mittel geeignet erscheinen, um bestimmte Ziele zu erreichen. Eine solche Annahme könnte in unserem Beispiel unter anderem sein: Damit Jugendliche zu einer kritischen Reflexion ihrer eigenen Computerspielnutzung kommen, sollen sie aus geeigneten Texten Informationen zu Einflüssen von Computerspielen und zu Jugendschutz-Bestimmungen in Kleingruppen erarbeiten und in der Klasse diskutieren.

Solche Merkmale und Vorstellungen verweisen auf verschiedene Anschlussmöglichkeiten an den medienpädagogischen Diskurs mit den oben genannten Themenfeldern, z. B. an den Diskurs zu Begriffen und zu zentralen Konstrukten, zu individuellen Voraussetzungen und Bedingungen sowie zu Zielvorstellungen und zu Vorgehensweisen. Allerdings setzt die Wahrnehmung dieser Anschlussmöglichkeiten ein reflexives Vorgehen aufseiten der praktisch Handelnden voraus. In diesem Zusammenhang kann man z. B. in Anlehnung an Schön (1983, 21-69) für den «Reflective Practitioner» drei Reflexionsmodi unterscheiden: Reflexionen in der Planungsphase von Handlungen, während des Handlungsvollzugs und nach der Handlung.

Demgemäss wäre es in dem oben skizzierten Beispiel wünschenswert, dass sich die Lehrperson vor dem Unterricht ihre diesbezügliche subjektive Theorie bewusstmacht und zu entsprechenden Themen des medienpädagogischen Diskurses in Beziehung setzt, sodass sie gegebenenfalls ihre Vorstellungen über einen wirksamen Unterricht modifizieren und in die Planung einbringen könnte. Weiterhin wäre erstrebenswert, dass die Lehrperson bei der Unterrichtsdurchführung kritische Situationen kurzfristig analytisch erfasst und gegebenenfalls vor dem Hintergrund ihres 
medienpädagogischen Wissens ihr Handeln der jeweiligen Situation anpasst. Und schliesslich sollte die Lehrperson im Anschluss an die Unterrichtssituation gelungene, problematische, erwartungswidrige oder überraschende Aspekte bzw. Situationen handlungsentlastet in den Blick nehmen und auf der Grundlage ihres medienpädagogischen Wissens mögliche Handlungsalternativen bedenken (vgl. Tulodziecki et al. 2017, 328).

Bezogen auf das Thema Computerspiel könnte die Lehrperson durch entsprechende Reflexionen schon bei der Planung des Unterrichts darauf aufmerksam werden, dass die mangelnde Bereitschaft von Jugendlichen, das eigene Computerspielverhalten zu reflektieren, nicht unbedingt damit zusammenhängt, dass gegebenenfalls Kenntnisse zu Medieneinflüssen oder zu Jugendschutz-Bestimmungen fehlen, sondern eher darauf zurückzuführen ist, dass die Nutzung von Computerspielen mit der Befriedigung von Bedürfnissen nach Spannung, Kompetenz oder Zugehörigkeit verbunden ist (oder zumindest sein kann) und insofern positive Emotionen hervorruft, die sich Jugendliche nicht gerne streitig machen lassen (vgl. z. B. Tulodziecki et al. 2010, 48-52). Des Weiteren könnte die Lehrperson bei Einbezug des medienpädagogischen Diskurses erfahren haben, dass Interesse an einem Thema in der Regel nicht dadurch geweckt wird, dass Informationen erarbeitet werden müssen, sondern eher dadurch, dass ein interessantes Problem oder ein bedeutsamer Entscheidungsfall oder eine für Jugendliche relevante Gestaltungs- oder Beurteilungsaufgabe als Ausgangspunkt für unterrichtliche Aktivitäten dient. Beobachtet die Lehrperson dann im Verlauf des Unterrichts beispielsweise, dass Jugendliche versuchen, sich der Auseinandersetzung mit einem an sich bedeutsamen Fall zu entziehen, kann sie mit Möglichkeiten gegensteuern, wie sie ebenfalls in der medienpädagogischen Literatur zu finden sind. Darüber hinaus kann die Lehrperson den medienpädagogischen Diskurs in einer anschliessenden Reflexion nutzen, um z. B. bei weniger gelungenen Passagen des Unterrichts auf Alternativen für ein zukünftiges Vorgehen aufmerksam zu werden (vgl. zu einem entsprechenden Unterricht ebd., 319-338).

Insgesamt stellt ein solchermassen reflexives Handeln mit der Fähigkeit, situationsbezogene Anforderungen mit medienpädagogischem bzw. wissenschaftlichem Wissen in Beziehung zu setzen, ein wichtiges Merkmal medienpädagogischer bzw. pädagogischer Professionalität dar. Bezogen auf entsprechende Zusammenhänge fordert beispielsweise Benner $(1980,485)$ von der Erziehungswissenschaft generell eine Sprache, die «Wissenschaft und Praxis in eine Beziehung setzt, welche Sorge dafür trägt, dass die Praxis aus der Wissenschaft Aufklärung, Orientierung und Kritik und die Wissenschaft aus der Praxis Erfahrung, Anregung und Korrektur ihrer Theoriebildung gewinnen kann». Dabei ist allerdings zu beachten, dass der medienpädagogische Diskurs - wie auch andere erziehungswissenschaftliche Diskurse - mittlerweile so komplex und umfangreich geworden ist, dass er weder in der Ausbildung noch in der Fort- oder Weiterbildung oder autodidaktisch aus der praktischen Arbeit 
heraus in all seinen Facetten erschlossen werden kann. Es werden immer nur exemplarische Erschliessungen möglich sein.

Vor einem solchen Hintergrund ergeben sich zwei Folgerungen zur Frage nach dem Verhältnis von Theorie und Praxis:

1. Das Theorie-Praxis-Verhältnis kann weder mit einem einfachen Transfermodell, nach dem sich Theorie unmittelbar auf Praxis übertragen lässt, noch mit dem so genannten Transformationsmodell, nach dem man Theorie durch bestimmte Transformationsregeln in Handlungsanleitungen umformen kann, die sich dann anwenden lassen, angemessen beschrieben werden. Vielmehr ist das TheoriePraxis-Verhältnis als ein situationsbezogenes In-Beziehung-Setzen von subjektiver Theorie und wissenschaftlicher Theorie in Handlungszusammenhängen zu verstehen, bei dem subjektive Theorie modifiziert und im Kontext der Analyse und Gestaltung praktischer Situationen rekonstruiert wird (vgl. Patry 2012, 9-15; Tulodziecki et al. 2017, 319-322).

2. Die Umsetzung eines solchen Theorie-Praxis-Verhältnisses könnte durch fundierte und verständlich geschriebene Einführungen und Übersichten, die einen guten Anschluss an praktische Situationen und Anforderungen ermöglichen, erleichtert werden. Des Weiteren liessen sich entsprechende Prozesse durch elektronische Zusammenstellungen mit einem geeigneten Verweis- und Suchsystem unterstützen. Damit zeichnen sich wichtige Anforderungen an diejenigen ab, die sich mit ihren Kommunikationsbeiträgen am medienpädagogischen Diskurs beteiligen.

Im Zusammenhang mit der Komplexität des medienpädagogischen Diskurses ergibt sich des Weiteren die Frage, ob und wie er gegebenenfalls begrenzt werden kann, ohne seine Wirksamkeit für Erkenntnisgewinn und Handeln zu verlieren.

\section{Zur Reduzierung von Komplexität im medienpädagogischen Diskurs durch Grenzzie-} hungen

Bei der Frage nach einer Begrenzung des medienpädagogischen Diskurses liegt erneut eine Bezugnahme auf systemtheoretische Ansätze nahe. In unserem Zusammenhang ziehe ich die folgenden systemtheoretischen Annahmen heran:

a. Nach Luhmann $(1984,45-57)$ besteht eine Hauptfunktion von Systembildungen in der Reduzierung von Komplexität. Diese kann dadurch erreicht werden, dass wie oben bereits angesprochen - zwischen einem System und seiner Umwelt unterschieden wird. Mit der Unterscheidung erfolgt eine Grenzziehung, die zugleich die Möglichkeit eröffnet, innerhalb des Systems eine höhere Ordnung zu schaffen als in der Systemumwelt.

b. Soziale Systeme lassen sich als autopoietische Systeme interpretieren, was bedeutet, dass sie sich in der Kommunikation selbst reproduzieren und gegebenenfalls auch neu produzieren (vgl. Luhmann 1984, 57-70). Demgemäss können 
sich Kommunikationsereignisse im Diskurs auf Vorheriges beziehen und dies bei anschliessenden Kommunikationen berücksichtigen. In diesem Sinne besitzen soziale Systeme «Reflexivität» und sind insofern selbstbezüglich bzw. selbstreferentiell.

c. Parsons $(2009,12)$ schreibt Handlungssystemen vier zentrale Funktionen zu, die für das Fortbestehen und die Weiterentwicklung von Systemen bedeutsam sind: Integration, Normenerhaltung (gekoppelt mit schöpferischem Normenwandel), Zielverwirklichung und Anpassung. Verallgemeinert man entsprechende Überlegungen, ergibt sich auch für Diskurse die Frage, inwieweit Integration, hinreichende Normenerhaltung und Zielerreichung bei gegebenenfalls notwendigem Normenwandel und notwendiger Anpassung gesichert werden können.

Aus diesen drei systemtheoretischen Annahmen ergeben sich zur Frage der Reduzierung von Komplexität im medienpädagogischen Diskurs verschiedene schlussfolgernde Überlegungen:

1. Bei einem Verständnis des medienpädagogischen Diskurses als soziales System, das sich zur Reduzierung von Komplexität und zur Entwicklung «innerer Ordnung» von seiner Systemumwelt abgrenzen muss, können - in idealtypischer Denkweise - alle Kommunikationsereignisse aus dem Diskurs herausgehalten werden, die keine Erziehungs- oder Bildungsrelevanz mit Medienbezug aufweisen, z. B. rein psychologische, soziologische, informatische oder rein medien- bzw. kommunikationswissenschaftliche Betrachtungen. Damit ist allerdings das - schon eingangs thematisierte - Problem verbunden, dass es bei einer entsprechenden Grenzziehung durchaus strittig sein kann, was im Detail unter Erziehungs- und Bildungsrelevanz sowie unter Medienbezug zu verstehen ist und was als wichtige Voraussetzung oder Bedingung für entsprechende Aktivitäten zu gelten hat. Mit diesem Problem kann jedoch auch der Vorteil verbunden sein, dass wichtige Überlegungen aus der Systemumwelt nicht vorschnell «abgeblockt», sondern auf ihre Anschlussfähigkeit geprüft werden

2. Aus der Sichtweise eines autopoietischen bzw. selbstreferentiellen Systems hat auch der medienpädagogische Diskurs eine «Geschichte», die auf die jeweilige aktuelle Kommunikation zurückwirkt. Des Weiteren folgt daraus, dass grundsätzlich nur das wahrgenommen bzw. aufgenommen wird, was anschlussfähig an die Kommunikation innerhalb des Systems ist oder in der Kommunikation anschlussfähig gemacht werden kann. Dies würde bedeuten, dass z. B. aus Diskursen in Philosophie, Psychologie, Soziologie, Informatik und Medien- bzw. Kommunikationswissenschaft nur das Eingang in medienpädagogische Überlegungen finden kann, was an erziehungs- und bildungsbezogene Erwägungen zu Medienfragen anknüpft oder in eine anschliessbare Form gebracht wird. Dies hiesse aber auch, dass unter Umständen wichtige Gedanken, z. B. aus der Informatik 
oder der Kommunikationswissenschaft, keinen Eingang in den medienpädagogischen Diskurs finden, weil ihnen (zunächst) die Anschlussfähigkeit fehlt.

3. Im Hinblick auf das Fortbestehen oder die Weiterentwicklung der Medienpädagogik ergeben sich aus den Überlegungen zum Systemerhalt beispielsweise die Fragen, ob und wie neuere Überlegungen - etwa zur Digitalisierung oder zu «Big Data» - in den medienpädagogischen Diskurs integriert werden können, welche Normen, z. B. bisherige Orientierungen an Medienkompetenz oder Medienbildung, erhalten bleiben oder verändert werden müssen, ob und inwieweit die Ziele bzw. Intentionen von Medienpädagogik, praktisches Erziehungs- und Bildungshandeln in Medienzusammenhängen aufzuklären und gleichzeitig Anregungen und Unterstützungen zur Gestaltung eines entsprechenden Handelns zu geben, erreicht werden können. Mit solchen Integrations-, Normen- und Zielüberlegungen sind zugleich Fragen nach der Notwendigkeit von Anpassungen verbunden, z.B. hinsichtlich der Kompetenzanforderungen für medienpädagogisch Tätige oder hinsichtlich bildungspolitischer Forderungen nach einer so genannten «digitalen Bildung». «Umgekehrt» stellt sich die Frage, wie die Systemumwelt des medienpädagogischen Diskurses, z. B. der bildungspolitische Diskurs, durch Rückwirkungen des medienpädagogischen Diskurses beeinflusst werden kann. Diese drei Überlegungen verweisen mit aller Deutlichkeit darauf, dass die Medienpädagogik vor der ständigen Herausforderung steht, über eine Balance von Grenzziehung und Öffnung, z. B. zu anderen Diskursen, ihren Ansprüchen auf wissenschaftlich fundierte Aufklärung und Reflexion sowie ihrer Intention nach wissenschaftlich basierter Gestaltung von Erziehung und Bildung in Medienzusammenhängen gerecht zu werden. Als ein aktuelles Beispiel für diese Herausforderung kann die gegenwärtige Diskussion um das Verhältnis von Medienbildung und informatischer Bildung gelten (vgl. u. a. Rummler et al. 2016).

Zieht man hinsichtlich möglicher Weiterentwicklungen ergänzende Überlegungen heran, z. B. zu einer Kombination system- und evolutionstheoretischer Ansätze, ist im medienpädagogischen Diskurs eine Verbindung von Variation, Selektion und Stabilisierung anzustreben (vgl. Scheunpflug 1999, 172-173). Dabei werden unter Variation neuartige, von der bisherigen Struktur abweichende Ereignisse verstanden, Selektion meint die Eliminierung variationsbedingter Überschüsse und Stabilisierung bezieht sich auf die dauerhafte Beibehaltung selektierter Varianten. In diesem Sinne ist zu erhoffen, dass es der Medienpädagogik auch in Zeiten, in denen Digitalisierung in aller Munde ist, gelingt, einerseits neuartige Ansätze zu berücksichtigen, sich andererseits aber nicht in kommunikationsbezogenen «Überschüssen» zu verlieren, sondern diese unter der Erziehungs- und Bildungsperspektive so zu selektieren und gegebenenfalls zu stabilisieren, dass ein weiterentwickeltes wissenschaftlich-medienpädagogisches Gebilde Bestand haben kann. 


\section{Literatur}

Aßmann, Sandra, Bardo Herzig, und Gerhard Tulodziecki. 2016. Medienpädagogische Begriffsund Theoriebildung im Wandel - ein diskursanalytischer Zugriff. Abstract für die Publikation zur Jahrestagung der DGfE-Kommission Wissenschaftsforschung «Theorien und Theorieentwicklungen (in) der Erziehungswissenschaft» im Herbst 2016 an der Universität Erfurt.

Ausubel, David P. 1978. «Die Förderung bedeutungsvollen verbalen Lernens». Unterrichtswissenschaft 6 (1): 58-66.

Benner, Dietrich. 1980. «Das Theorie-Praxis-Verhältnis in der Erziehungswissenschaft und die Frage nach den Prinzipien pädagogischen Denkens und Handelns». Zeitschrift für Pädagogik 26 (4), 485-497.

Benner, Dietrich. 2012. Allgemeine Pädagogik. Eine systematisch-problemgeschichtliche Einführung in die Grundstruktur pädagogischen Denkens und Handelns. 7. Auflage. Weinheim u. München: Beltz.

Bettinger, Patrick, und Sandra Aßmann. 2017. "Das diskursive Feld um Mediatisierung und Mediensozialisation. Eine Analyse deutschsprachiger Fachzeitschriften». In Mediatisierung und Mediensozialisation, herausgegeben von Dagmar Hoffmann, Friedrich Krotz, und Wolfgang Reißmann, 79-100. Wiesbaden: Springer Fachmedien Wiesbaden. doi:10.1007/978-3658-14937-6_5.

Breiter, Andreas, Stefan Welling, und Björn E. Stolpmann. 2010. Medienkompetenz in der Schule: Integration von Medien in den weiterführenden Schulen in Nordrhein-Westfalen. Herausgegeben von Landesanstalt für Medien Nordrhein-Westfalen. Berlin: VISTAS Verlag. https:// www.lfm-nrw.de/fileadmin/lfm-nrw/Forschung/LfM-Band-64.pdf.

Budde, Jürgen. 2012. «Die Rede von der Heterogenität in der Schulpädagogik. Diskursanalytische Perspektiven». Forum: Qualitative Sozialforschung 13 (2), Art. 16.

Eickelmann, Birgit. 2010. Digitale Medien in Schule und Unterricht erfolgreich implementieren. Eine empirische Analyse aus der Sicht der Schulentwicklungsforschung. Münster: Waxmann.

Foucault, Michel. 1981. Archäologie des Wissens. Frankfurt a. M.: Suhrkamp.

Gross, Friederike von, Dorothee M. Meister, und Uwe Sander (Hrsg.). 2015. Medienpädagogikein Überblick. Weinheim u. München: Beltz Juventa.

Gysbers, Andre. 2008. Lehrer - Medien - Kompetenz. Eine empirische Untersuchung zur medienpädagogischen Kompetenz und Performanz niedersächsischer Lehrkräfte. Berlin: VISTAS.

Hartung, Anja, Bernd Schorb, Horst Niesyto, Heinz Moser, und Petra Grell, Hrsg. 2014. Jahrbuch Medienpädagogik 10. Methodologie und Methoden medienpädagogischer Forschung. Bd. 10. Jahrbuch Medienpädagogik. Wiesbaden: Springer Fachmedien Wiesbaden. http:// link.springer.com/10.1007/978-3-658-04718-4.

Herzig, Bardo, Dorothee M. Meister, Heinz Moser, und Horst Niesyto, Hrsg. 2010. Jahrbuch Medienpädagogik 8. Medienkompetenz und Web 2.0. Bd. 8. Jahrbuch Medienpädagogik. Wiesbaden: VS Verlag für Sozialwissenschaften. http://link.springer.com/10.1007/978-3-53192135-8. 
Hüther, Jürgen, und Bernd Schorb, Hrsg. 2005. Grundbegriffe Medienpädagogik. 4. Auflage. München: kopaed.

Jörissen, Benjamin, und Winfried Marotzki. 2009. Medienbildung - eine Einführung: Theorie Methoden - Analysen. Bad Heilbrunn: Klinkhardt/UTB.

König, Eckard, und Gerda Volmer. 2008. Handbuch Systemische Organisationsberatung. Weinheim: Beltz.

Luhmann, Niklas. 1984. Soziale Systeme. Grundriss einer allgemeinen Theorie. Frankfurt a. M. Suhrkamp.

Moser, Heinz, Petra Grell, und Horst Niesyto, Hrsg. 2011. Medienbildung und Medienkompetenz: Beiträge zu Schlüsselbegriffen der Medienpädagogik. Bd. 20 Medienbildung im Spannungsfeld medienpädagogischer Leitbegriffe. München: Kopaed. http://www.medienpaed.com/ issue/view/29.

Parsons, Talcott. 2009. Das System moderner Gesellschaften. 7. Auflage. Weinheim u. München: Beltz Juventa.

Patry, Jean-Luc. 2012. «Der Pädagogische Takt». In Enzyklopädie Erziehungswissenschaft Online; Fachgebiet: Schulpädagogik, herausgegeben von Sibylle Rahm und Christian Nerowski. Weinheim u. Basel: Beltz Juventa. doi:10.3262/EEO 09120250.

Rummler, Klaus, Beat Döbeli Honegger, Heinz Moser, und Horst Niesyto, Hrsg. 2016. Medienbildung und informatische Bildung - quo vadis? Bd. 25. Themenheft. Zürich: MedienPädagogik: Zeitschrift für Theorie und Praxis der Medienbildung. doi:10.21240/mpaed/25.X.

Sander, Uwe, Friederike von Gross, und Kai-Uwe Hugger, Hrsg. 2008. Handbuch Medienpädagogik. Wiesbaden: VS Verlag für Sozialwissenschaften.

Scheunpflug, Annette. 1999. «Evolutionäre Didaktik. Ein Entwurf aus system- und evolutionstheoretischer Sicht». In Neue Wege in der Didaktik? Analysen und Konzepte zur Entwicklung des Lehrens und Lernens, herausgegeben von Heinz Günter Holtappels und Marianne Horstkemper, 169-185, 5. Beiheft der Zeitschrift Die Deutsche Schule. Weinheim: Juventa.

Schön, Donald A. 1983. The Reflective Practitioner: How Professionals Think in Action. New York: Basic Books, Inc.

Schulz-Zander, Renate, Birgit Eickelmann, Heinz Moser, Horst Niesyto, und Petra Grell, Hrsg. 2012. Jahrbuch Medienpädagogik 9. Bd. 9. Jahrbuch Medienpädagogik. Wiesbaden: VS Verlag für Sozialwissenschaften. http://link.springer.com/10.1007/978-3-531-94219-3.

Schurz, Gerhard. 2014. "Koexistenz und Komplementarität rivalisierender Paradigmen: Analyse, Diagnose und kulturwissenschaftliches Fallbeispiel». In Die multiparadigmatische Struktur der Wissenschaften, herausgegeben von Stephan Kornmesser und Gerhard Schurz, 47-62. Wiesbaden: Springer VS.

Sesink, Werner, Michael Kerres, und Heinz Moser, Hrsg. 2007. Jahrbuch Medienpädagogik 6. Medienpädagogik- Standortbestimmung einer erziehungswissenschaftlichen Disziplin. Bd. 6. Jahrbuch Medienpädagogik. Wiesbaden: VS Verlag für Sozialwissenschaften. http:// www.springer.com/de/book/9783531153643.

Spanhel, Dieter. 2006. Medienerziehung. Erziehungs- und Bildungsaufgaben in der Mediengesellschaft. Stuttgart: Klett-Cotta. 
Swertz, Christian. 2008. «Hinweise zu einer Theorie der Medienpädagogik». In Medienbildung in Österreich. Historische und aktuelle Entwicklungen, theoretische Positionen und Medienpraxis, herausgegeben von Edith Blaschitz und Martin Seibt, 42-51. Wien, Berlin u. Münster: Lit.

Tulodziecki, Gerhard, Bardo Herzig, und Sigrid Blömeke. 2017. Gestaltung von Unterricht. Eine Einführung in die Didaktik. 4. Auflage. Bad Heilbrunn: Klinkhardt/UTB.

Tulodziecki, Gerhard, Bardo Herzig, und Silke Grafe. 2010. Medienbildung in Schule und Unterricht. Grundlagen und Beispiele. Bad Heilbrunn: Klinkhardt/ UTB.

Tulodziecki, Gerhard, Silke Grafe, und Bardo Herzig. 2013. Gestaltungsorientierte Bildungsforschung und Didaktik. Theorie - Empirie - Praxis. Bad Heilbrunn: Klinkhardt.

Tulodziecki, Gerhard. 2015. «Medienkompetenz». In Medienpädagogik - ein Überblick, herausgegeben von Friederike von Gross, Dorothee M. Meister und Uwe Sander, 194-228. Weinheim u. Basel: Beltz Juventa. 